\title{
Antimicrobial Surface Functionality of PEG Coated and AgNPs Immobilized Extracorporeal Biomaterials
}

\author{
Fikret Karademir 1 1D , Fatma Ayhan 2,* (D) \\ 1 Muğla Sitkı Koçman University, Vocational School of Health Services, Marmaris, Muğla, Turkey; karademir@mu.edu.tr \\ (F.K.); \\ 2 Muğla Sitkı Koçman University, Faculty of Science, Department of Chemistry, Biochemistry Division, \\ (Biochemistry\&Biomaterials Research Group, BIOMATREG) Muğla, Turkey; fayhan@mu.edu.tr (F.A.); \\ * Correspondence: fayhan@mu.edu.tr;
}

Scopus Author ID 36842986600

Received: 23.01.2021; Revised: 10.04.2021; Accepted: 14.04.2021; Published: 26.04.2021

\begin{abstract}
Nanotechnology is a promising field for generating new medicine applications like colloidal silver to prevent nosocomial infections. In this study, the objection of the presented manuscript was to test the antimicrobial activity of PEG/silver nanoparticles (AgNPs) coated blood tubing used in hemodialysis. The modification of plastic tubing was first performed by PEG coating, which was followed by immobilization of AgNPs synthesized with a green chemical approach from the leaves of Liquidambar Orientalis Miller. The stabilization of each surface modification stage was assured by methanol fixation. The characterization of AgNPs, surface modification steps, and final tubing surface appearances was performed by spectrophotometric measurements, DLS analysis, FTIR spectrums, and SEM images. The results indicated that the surfaces of hemodialysis tubing were successfully coated with PEG/AgNPs processed sequentially. Antimicrobial activities were evaluated against pathogens (Escherichia coli, Pseudomonas aeruginosa. Klebsiella pneumonia, Staphylococcus aureus, and Candida albicans), which are commonly involved in catheter-related infections. In conclusion, a new AgNPs immobilization method to polymeric catheter surfaces after PEG layering was developed. The antimicrobial function against five nosocomial microorganisms would have potential in biomedical applications.
\end{abstract}

Keywords: medical polymers; macromolecular reactions; nanoparticles; immobilization; antimicrobial properties.

(C) 2021 by the authors. This article is an open-access article distributed under the terms and conditions of the Creative Commons Attribution (CC BY) license (https://creativecommons.org/licenses/by/4.0/).

\section{Introduction}

Intravascular catheters, or blood tubings, which are one of the indispensable instruments in modern medicine, are applied intravascular in different units, notably in intensive care units for specific reasons (drugs, blood products, electrolytes, fluids, provision of parenteral foods, total parenteral diet; hemodynamic monitoring; blood sample taken for diagnostic purposes and hemodialysis application, etc.) and can be used for a long time. Although catheters provide a lot of benefits for patients with this wide-range of beneficial usages, they lead to significant levels of mortality and morbidity both for mechanical reasons (pneumothorax, hemothorax, thrombus, emboli, and fistula formation) and due to infectionrelated complications [1].

Thrombus formation and infection, especially due to bacteremias and bacterial colonization that could provoke severe diseases, are the most significant complications of long- 
term and permanent catheter use [2-6]. The most commonly encountered factors of catheter colonization are microorganisms that can form biofilms, such as coagulase-negative staphylococci, Staphylococcus aureus, Pseudomonas aeruginosa, and Candida species. Therefore, pathogenic problems need new strategies to prevent implant-associated infections [7-11].

Biomaterial-associated infections are caused by attached pathogenic cells, and bioactive coatings have an attraction to overcome this problem. The designed bioactive coatings on material surfaces can inhibit bacterial adhesion and eliminate the attached pathogenic cells [12-14]. Studies to render the catheters antimicrobial continue, especially on catheter surface modifications $[2,3,9,10,15,16]$. The most significant known property of silver nanoparticles is prevention against infections [17-19]. The fact that silver nanoparticles, between 1 and $100 \mathrm{~mm}$, can be sized down to such dimensions dramatically increases its destruction and long protection activation on bacteria, viruses, and fungi. The oxidation of silver nanoparticles to silver ions and change the driving force for ATP synthesis by electrochemical proton gradient through respiratory processes in bacteria were proposed as possible antibacterial mechanisms [20,21].

Catechol group of the polydopamine film was used to generate AgNPs on PTFE, central venous catheters, to prevent catheter-related bloodstream infections [8]. The deposition of silver nanoparticles on standard PE-BAX polyamide 20 gauge catheters was performed using $\mathrm{N}, \mathrm{N}, \mathrm{N}$ o,N o-tetramethylethylenediamine as a reducing agent [20]. The coating of catheters (tubes that can be placed in the body cavity, veins, and channels) surfaces with silver nanoparticles, [22-24] the materials gain antimicrobial characteristics [25-27]. Silver nanoparticle deposition on Standard PE-BAX polyamide 20 gauge catheters was performed by forming nanoparticles by AcryMed SilvaGard technology (published US patent application 20070003603) where a surfactant and N,N,N o,N o-tetramethylethylenediamine were used as a reducing agent. Coated catheters showed significant in vitro antimicrobial activity and prevented biofilm formation using Escherichia coli, Enterococcus, Staphylococcus aureus, coagulase-negative staphylococci, Pseudomonas aeruginosa, and Candida albicans [25,28]. Inspired by mussel adhesive proteins, dopamine was used to form a thin polydopamine layer and induce AgNPs formation without additional reductants or stabilizers. The chemical and physicochemical properties of AgNPs coated CVCs were characterized by scanning electron microscopy, X-ray photoelectron spectroscopy, Raman spectroscopy, and water contact angle. The Staphylococcus aureus culture experiment was used to study the antibacterial properties [8]. PET film surfaces were modified with anti-adhesive and antibacterial materials, heparin and chitosan, respectively. Aminated PET surfaces were treated to form multilayer heparin/chitosan films by alternate polyelectrolyte deposition [13, 29].

However, the advantage of antimicrobial properties of biocompatible material PEG and green synthesized [30] silver nanoparticle coated catheters have not been investigated. The studies presented in this report was studies presented this report aimed to develop a new method to deposit PEG and then silver nanoparticles, which were synthesized with an environmentallyfriendly approach on hemodialysis tubings to determine the antimicrobial activity of these catheters in vitro. The data suggest that catheter surfaces that have been modified to attain AgNPs and biocompatible macromolecule, PEG may effectively reduce the infectious risk associated with chronic catheterization in humans. 


\section{Materials and Methods}

\subsection{Materials.}

Silver nitrate $\left(\mathrm{AgNO}_{3}\right)$ was purchased from Merck and used as received without further purification. Siğla tree (Liquidambar orientalis Mill. Tree) leaves were obtained from Marmaris, Turkey. E. coli ATCC 25922; S. aureus ATCC25923; P. aeruginosa ATCC 27853 K. Pneumoniae ATCC13883; C. albicans ATCC 10231; methicillin-resistant S. aureus ATCC 29213 microbial strains were tested for their antimicrobial activities. Müller Hinton Agar [31] and Brain Heart Infusion broth [32] were purchased from Oxoid and Merck, respectively. Hemodialysis blood tubing sets were kindly supported by the local user in Turkey. Distilled water was used throughout the research.

\subsection{Synthesis and characterization of silver nanoparticles.}

In this study, leaves of Liquidambar Orientalis Miller var. integriloba (sweet gum) tree were collected from Marmaris National Park during September. After being washed to clear off the dirt on them, the collected leaves were left to dry on filter paper for approximately one week in an environment without direct sunlight exposure. The dried sweet gum leaves were put into a beaker, and ultra-pure water was added to prepare the concentration to be worked on. It was heated for 20 minutes and allowed to cool down thoroughly. The crude was then sieved with a filter, and extract with the desired concentration to be worked on was obtained. The silver nitrate solution used was prepared by adding pure water onto silver nitrate put into a volumetric flask to be used at different concentrations and ensuring that it was dissolved thoroughly. $10 \mathrm{~mL}$ of silver nitrate solution and $10 \mathrm{~mL}$ of sweet gum extract prepared were put into a beaker; $80 \mathrm{~mL}$ of pure water was added, so a general mixture was prepared. From this moment on, silver nanoparticle formation began, and the start of specified periods is the preparation moment of the mixture [33]. The fluid transfusion performed at previous and current stages was carried out with micropipette and graduated cylinders - the analysis of nanoparticle synthesis performed in Shimadzu UV-1201 spectrophotometer. Infrared Fourier Transformation spectrums of extracorporeal polymeric biomaterials before and after treatment were obtained using ATR-FTIR (Perkin Elmer, Spectrum 100). Spectrums were given as the mean of 25 measurements in a wavelength range of $4000-400 \mathrm{~cm}^{-1}$ at a resolution of $4 \mathrm{~cm}^{-1}$ resolutions. Dynamic Light Scattering (DLS) analysis was performed to estimate particle size distribution (Malvern, Zetasizer Ver. 6.0).

To examine the shape and dimensions of silver nanoparticles, transmission electron microscopy (TEM) measurements were performed on a JEOL model FEG-TEM device operated at an acceleration voltage of $200 \mathrm{kV}$.

\subsection{Surface modification of medical polymeric biomaterials.}

In the research, dialysis tubing was selected as an extracorporeal polymeric biomedical material. The surface of biomaterials was modified to immobilize AgNP via functional groups since the tubing used in dialysis was produced from medical grade PVC. The modification process was performed by soaking the biomaterial 2\% PEG2000 solution at room temperature and 250 strokes/ min for two hours. The coated surface was fixed with $70 \%$ methanol for 2 hours and the same temperature and shaking rate, and then methanol is discarded, and previously sonicated $0.5 \% \mathrm{AgNP}$ solution was added. The biomaterials were again treated with 
$70 \%$ methanol. The nanoparticle coating and fixation processes were prolonged for 2 hours. Table 1 gives the surface modification types applied to extracorporeal polymeric biomaterials, and the types of blood tubings were shown in Figure 1. The SEM images of untreated, PEGmodified, AgNP modified, and PEG+AgNP modified blood tubing surfaces were taken by scanning electron microscopy (SEM) using JEOL JSM-7600F SEM device.

Table 1. Surface Modifications of Extracorporeal Polymeric Biomaterials.

\begin{tabular}{l|l} 
Surface Modification Steps & Surface Properties \\
\hline 1. PVC $+2 \%$ PEG 2000 solution & PEG coated \\
\hline $2.1^{\text {st }}$ step $+70 \%$ MetOH solution & MetOH fixed \\
\hline $3.2^{\text {nd }}$ step $+0,5 \%$ AgNP solution & AgNPs immobilized \\
\hline $4.3^{\text {rd }}$ step $70 \%$ MetOH solution & MeOH Fixed
\end{tabular}

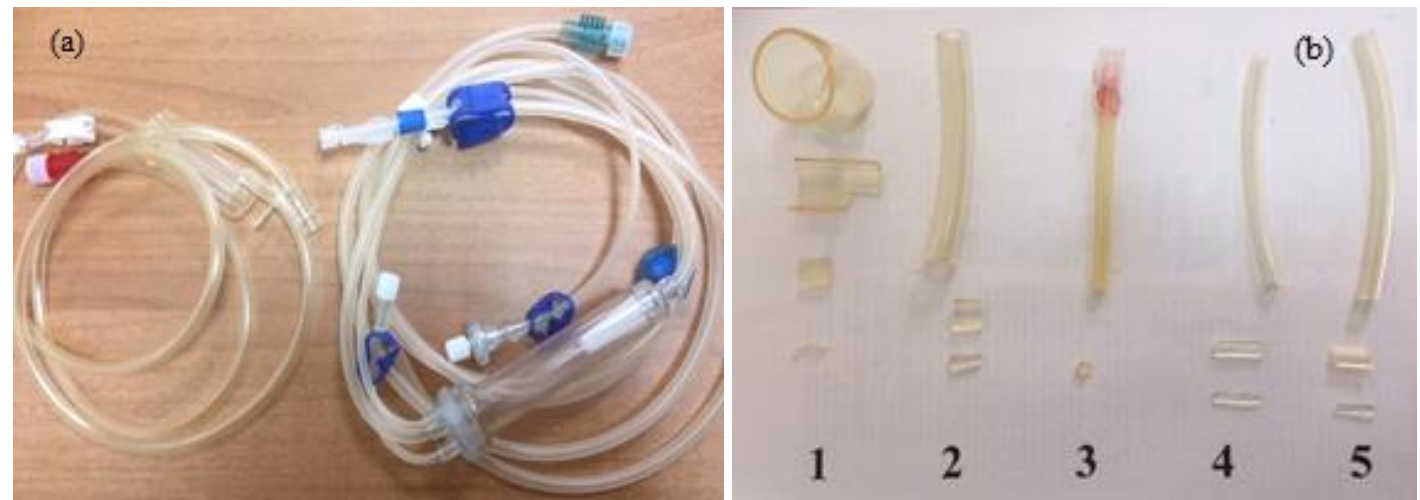

Figure 1. a) Arterial and venous dialysis blood tubings, b) Types of dialysis tubings with different wall thicknesses, 1) $1.8 \mathrm{~mm}, 2) 1.5 \mathrm{~mm}, 3) 1.3 \mathrm{~mm}$, 4) $1.2 \mathrm{~mm}, 5) 0.5 \mathrm{~mm}$ and their corresponding parts tested.

\subsection{Antimicrobial activity tests.}

Antibacterial activities of modified extracorporeal polymeric biomaterials have been tested by the Disc Diffusion method (modified) [34] and AATCC 100-2007 standard. Inoculated microorganisms were prepared by growing cells in Nutrient broth (Merck) [35]. For $24 \mathrm{~h}$ at $37^{\circ} \mathrm{C}$. These cell suspensions were diluted with peptone water to provide initial cell counts of about $10^{5}-10^{6} \mathrm{CFU} / \mathrm{mL}$. $17 \mathrm{~mL}$ sterile Muller Hinton agar at $45^{\circ} \mathrm{C}$ and poured into Petri dishes $\left(9 \mathrm{~cm}\right.$ in diameter). Then the agars were allowed to solidify at $4^{\circ} \mathrm{C}$ for $1 \mathrm{~h}$. Test microorganisms were spread on Muller Hinton agar. Sterile polymeric biomaterials with a dimension of $0.7 \mathrm{~cm}$ width and $1 \mathrm{~cm}$ length were placed in agar medium plates inoculated with microorganisms under sterile conditions. The inoculated Petri dishes were left 10-15 minutes at room temperature and then incubated $37^{\circ} \mathrm{C}$ for $24-48$ hours. After the incubation period was completed, inhibition zones were measured in millimeters. These experiments were carried out in duplicate [21]. Escherichia coli, Pseudomonas aeruginosa, Klebsiella pneumonia, Staphylococcus aureus, and Candida albicans were used as test microorganisms.

\section{Results and Discussion}

\subsection{Green synthesis and characterization of AgNPs.}

The sweetgum leaves, which were used as a reductant in the study, were collected from Marmaris National Park during the season (in September) from inner sections. The leaves became ready to be used after washed and dried one week at room temperature. It was observed that the vivid green appearance of leaves indicated that no disruption had occurred. After the 
dried leaves were pestled and powdered, a certain amount of leaves was boiled in water at $100^{\circ} \mathrm{C}$.

In the synthesis process, the color change in the solution with plant extract proves the synthesis of nanoparticles by visual examinations. The formation of metal nanoparticles through reducing silver ions exposed to plant extract was also followed with UV-Vis spectroscopy. The synthesis process was conducted at room temperature, and absorbance measurements were performed spectrophotometrically at the wavelength range of 300-800 nm by taking samples at specified time intervals. The readings were continued until nearly repetitive absorbance values were obtained, which was regarded as the termination of particle formation. The absorbance reading between $420-470 \mathrm{~nm}$ results from stimulation of surface plasmon vibrations of the reduction process of ionic metal [36]. UV-Vis spectra obtained during reduction (0-480 minutes) were given in Figure 2a.

DLS analysis was performed to study particle size and size distribution analysis of AgNPs synthesized using plant extract. DLS analysis results are given in Figure $2 \mathrm{~b}$. When DLS are examined, it is seen that AgNPs give a single $45 \mathrm{~nm}$-centered peak with a width of $20 \mathrm{~nm}$. The mean particle size was found as $48.89 \mathrm{~nm}$.
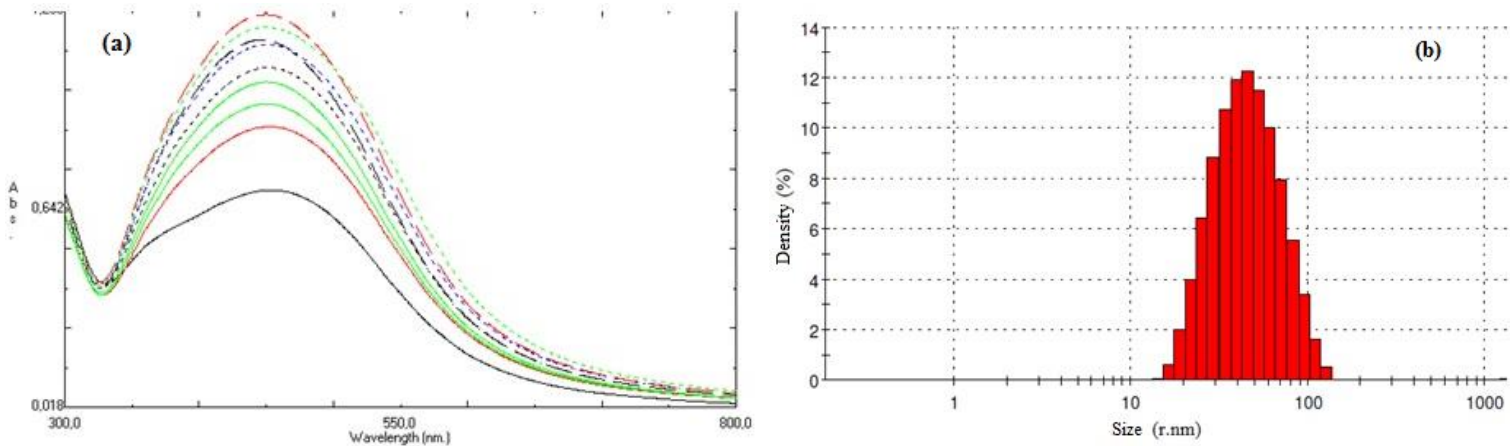

Figure 2. a) UV-Vis spectra of silver nanoparticles at various times, b) Particle size distribution analysis of AgNPs.

These results confirm that nanoparticles synthesized with plant extract have good stabilization. The results obtained from DLS analysis regarding particle size and distribution were found complementary with the results for peak width obtained from UV-Vis analysis since the width of spectra gives an opinion about the particle size distribution. The interaction of silver nanoparticles and human blood protein hemoglobin was studied. The formation of the nano-bio complex due to electron transfer mechanism has been dependent on nanoparticle size [37].

\subsection{Modifications of surface properties of polymeric biomaterials.}

The chemical structure PVC blood tubing and the functional groups in the chemical structure of PEG were depicted in Figures $3 \mathrm{a}$ and $3 \mathrm{~b}$.

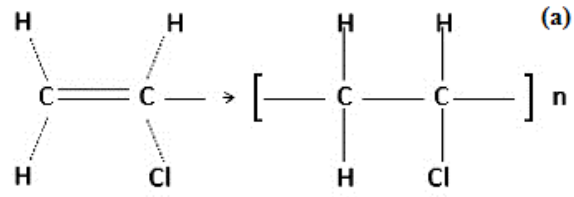

(a)<smiles>CC(C)CCC(C)O</smiles>

Figure 3. Chemical structures of a) Polyvinyl chloride (PVC), b) Polyethylene glycol PEG.

The blood-contacting polymeric biomaterial surfaces with different wall thicknesses and diameters were modified by treatment with PEG and green synthesized AgNPs solutions. 
The fixation of PEG and AgNPs were also applied for both surfaces. So, four types of modified surfaces were characterized for their functional group variations by FTIR spectrums given in Figure 4.

FTIR spectrum of untreated of 3rd type tubing of $5 \mathrm{~mm}$ outer diameter was given in Figure 4a. The peaks around 2930, 1720, and $1235 \mathrm{~nm}$ were mainly taken as reference peaks in order to compare with other spectrums of surface treatment processes. The spectrum of PEGtreated blood tubings was given in Figure 4b. Blood contacting tubing surfaces modified with PEG solution resulted in decreasing transmittance values of these peaks.

The characteristic peaks for PEG have been observed around at $3750-3640 \mathrm{~cm}^{-1}$ (hydroxyl group), $1730 \mathrm{~cm}^{-1}$ (hydrogen bonding), $1460 \mathrm{~cm}^{-1}\left(-\mathrm{CH}_{3}\right.$ and $-\mathrm{CH}_{2}$ bending), and $1140 \mathrm{~cm}^{-1}$ (anti-symmetric stretching) [38]. The intensity of the transmittance reduction range was observed between 5-10\% and accepted as an indication of the adsorption of PEG on tubing surfaces.
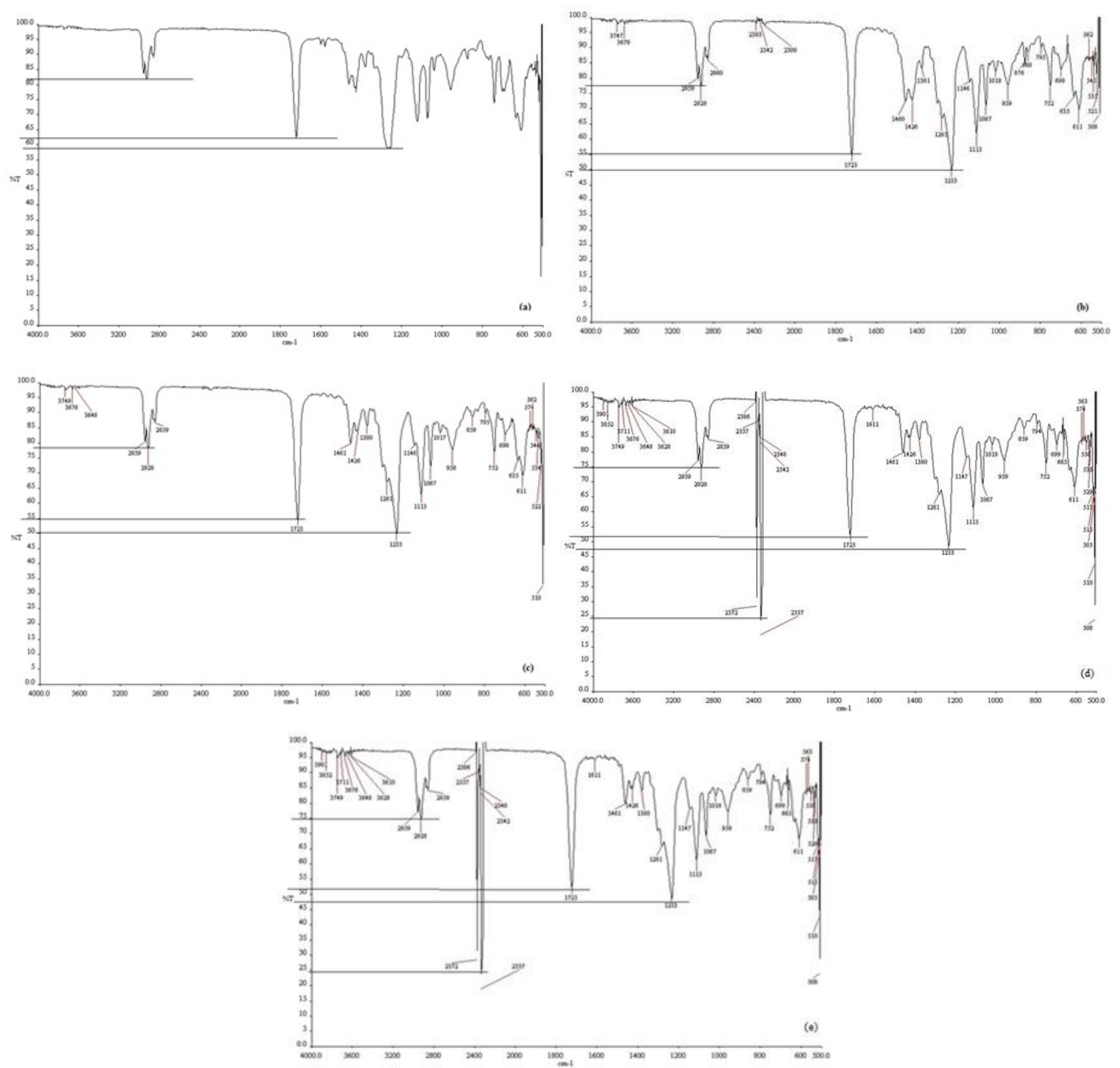

Figure 4. FTIR spectra of $2^{\text {nd }}$ Type Dialysis Tube: (a) Non-treated; (b) PEG2000, c) PEG2000+MetOH fixed d) PEG2000+MetOH fixed+AgNP, (e) PEG2000+MetOH fixed+AgNP+MetOH fixed.

Figure $4 \mathrm{c}$ shows the methanol fixation spectrum of dialysis tubing with the same diameter value after PEG treatment process. In general, small increments of all peaks were determined, while it can be seen from the intensity of the reference peaks. Additionally, this 
behavior can be seen at $2859 \mathrm{~cm}^{-1}, 2928 \mathrm{~cm}^{-1}$, and $2959 \mathrm{~cm}^{-1}$ peak values. Transmittance values at $2928 \mathrm{~cm}^{-1}, 1723 \mathrm{~cm}^{-1}$, and $1233 \mathrm{~cm}^{-1}$ were nearly 80,60 , and $50 \%$, while little increments in transmittance were observed after fixation with methanol.

The biomaterial surface treatment was continued with AgNPs coating following PEG and $\mathrm{MeOH}$ fixation processes.

The spectrums of AgNPs coated and then methanol fixed tubings were given in Figure $4 \mathrm{~d}$ and Figure 4e. The new sharp peaks appeared, and some peaks had higher intensity at peak values mentioned above.

The reference peak transmittances, 2930, 1720, and $1235 \mathrm{~cm}^{-1}$, decreased, which was interpreted as AgNP deposition on the biomaterial surface. More specifically, the peaks between $2348 \mathrm{~cm}^{-1}$ and $2386 \mathrm{~cm}^{-1}$ were regarded as carbon-halogen bending vibrations that may come from green synthesized AgNPs. The decrease of transmittance or intensity increment of the peaks corresponding to PEG functional groups may come from the interaction between PEG and AgNPs [39].

The surface treatment with $\mathrm{MeOH}$ after nanoparticle coating procedure resulted in an important decrease in abnormal peaks (Figure 4e.), protecting the peaks from PEG and AgNPs. $\mathrm{MeOH}$ fixation gave higher transmittance values to reference peaks, which indicates the formation of different surface properties.
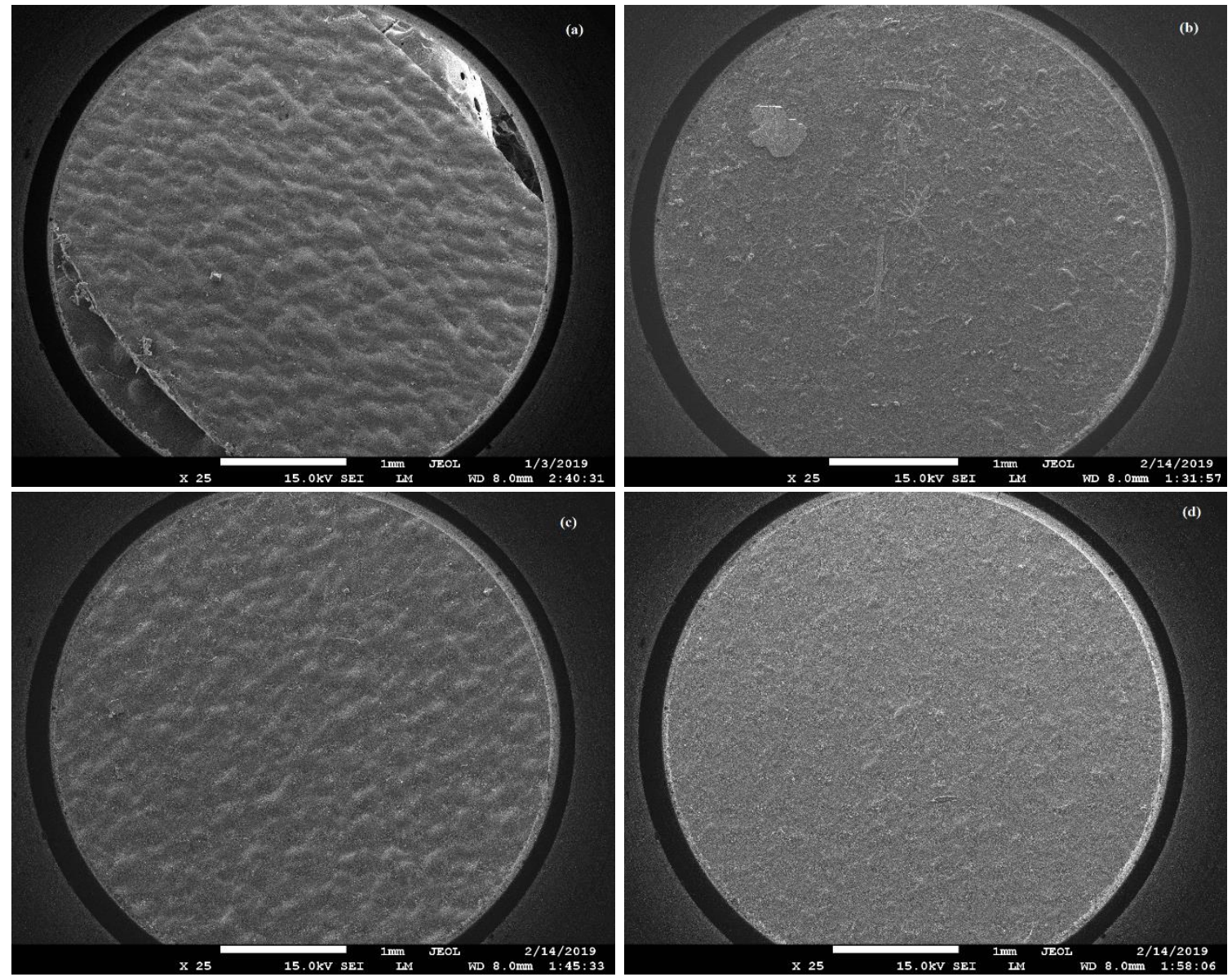

Figure 5. SEM images (x25) of a ) untreated PVC; b) PEG modified PVC; c) AgNP modified PVC; d) PEG + AgNP modified PVC surfaces.

SEM images of untreated and PEG, AgNP, and PEG+AgNP modified PVC surfaces were given in Figure 5 (x25 magnification). The untreated surface has a deep valley-like appearance, while it seems that PEG coating and/or accumulation resulted in the formation of a smoother surface after PEG immobilization step (Figure 5b). 
The AgNP treated biomaterial surface (Figure 5c) also has fewer holes than blank, which since the AgNP accumulation in valleys between hill-like structure. The PEG+AgNP image (Figure 5d) gives almost the total effect of two chemical processes, and the final surface has the most regular appearance.

Aggregation of AgNPs was also protected by PEG during synthesis or immobilization in the scaffold. So, the uniform deposition of AgNPs on the blood tubing surface was greatly realized $[40,41]$.

\subsection{Antimicrobial activity tests.}

In the initial stage of the research, blood tubings of $\sim 1 \mathrm{~cm}$ with different wall thicknesses and inner diameters were cut with a sharp knife to attain a smooth side by obtaining two similar shapes of tubings.

The $S$. aureus bacteria were inoculated on Muller Hinton Agar by spreading $10^{5}-10^{6}$ $\mathrm{CFU} / \mathrm{mL}$. Petri dishes were stored for $2 \mathrm{~h}$ at room temperature, and the biomaterials were placed so that their inner surfaces can touch the surface of growth media. After that, Petri dishes were incubated for $24+24 \mathrm{~h}$ at $37^{\circ} \mathrm{C}$ temperature and followed at certain times. Figure 6 shows the antibacterial activity of various tubing parts against $S$. aureus. The inhibition zones given in Table 2 are average antimicrobial inhibition diameters under the tubing materials. Figure 6 , the photographs of the tested biomaterials after $48 \mathrm{~h}$ incubation time were shown in Figure 6a, $6 \mathrm{c}$, and 6e. Modified tubing materials were gently removed, and their inhibited areas corresponding to the surface area of biomaterials were given in the right photos in Figure $6 \mathrm{~b}$, $6 \mathrm{~d}$, and 6e.

Due to the difficulty and complexity of the experimental application procedure explained above, the tubings were cut, such as to have parts $2 \mathrm{~mm}$ width and $1 \mathrm{~cm}$ length with a microtome in sterile conditions, and antimicrobial activities were analyzed against four bacterial and a fungal type nosocomial microorganisms. Figure 7 shows the antimicrobial activity results of five tubing parts given in Figure 1. The tubings were tested for antimicrobial activity after each surface coating process. The inhibition zone formations were almost the same for all of PEG+AgNp coated biomaterials for the tested shape. The antimicrobial activity data show that all microorganisms tested are sensitive to silver captured on the tubings. But sensitivity against microorganisms for the tubings with a small diameter is evidently less. These results greatly come from the contacted surface area treated with PEG+AgNPs is smaller than the dialysis tubings with a greater diameter.

On the other hand, it was reported that the stabilization of the hydroxyl group of PEG by covering the surface of AgNPs greatly occurs due to van der Waals forces between negative PEG and a positive charge on AgNPs [29]. The capping behavior of PEG may prevent the exhibition of antimicrobial activities of nanoparticles.

Table 2. Inhibition zones forming in $\mathrm{MH}$ agar.

\begin{tabular}{|l|c|c|c|c|c|}
\hline \multirow{2}{*}{ M.O Sample no } & \multicolumn{5}{|c|}{ Inhibition zone (mm) } \\
\cline { 2 - 6 } & $\mathbf{1}$ & $\mathbf{2}$ & $\mathbf{3}$ & $\mathbf{4}$ & $\mathbf{5}$ \\
\hline \hline E. coli & 4,0 & 5,0 & 8,0 & 4,0 & 4,0 \\
\hline P. aeruginosa & 4,0 & 5,0 & 9,0 & 7,0 & 5,0 \\
\hline K. pneumoniae & 5,0 & 3,0 & 9,0 & 6,0 & 3,0 \\
\hline S. aureus & 4,0 & 4,0 & 14,0 & 5,0 & 4,0 \\
\hline C. albicans & 4,0 & 5,0 & 4,0 & 8,0 & 7,0 \\
\hline
\end{tabular}



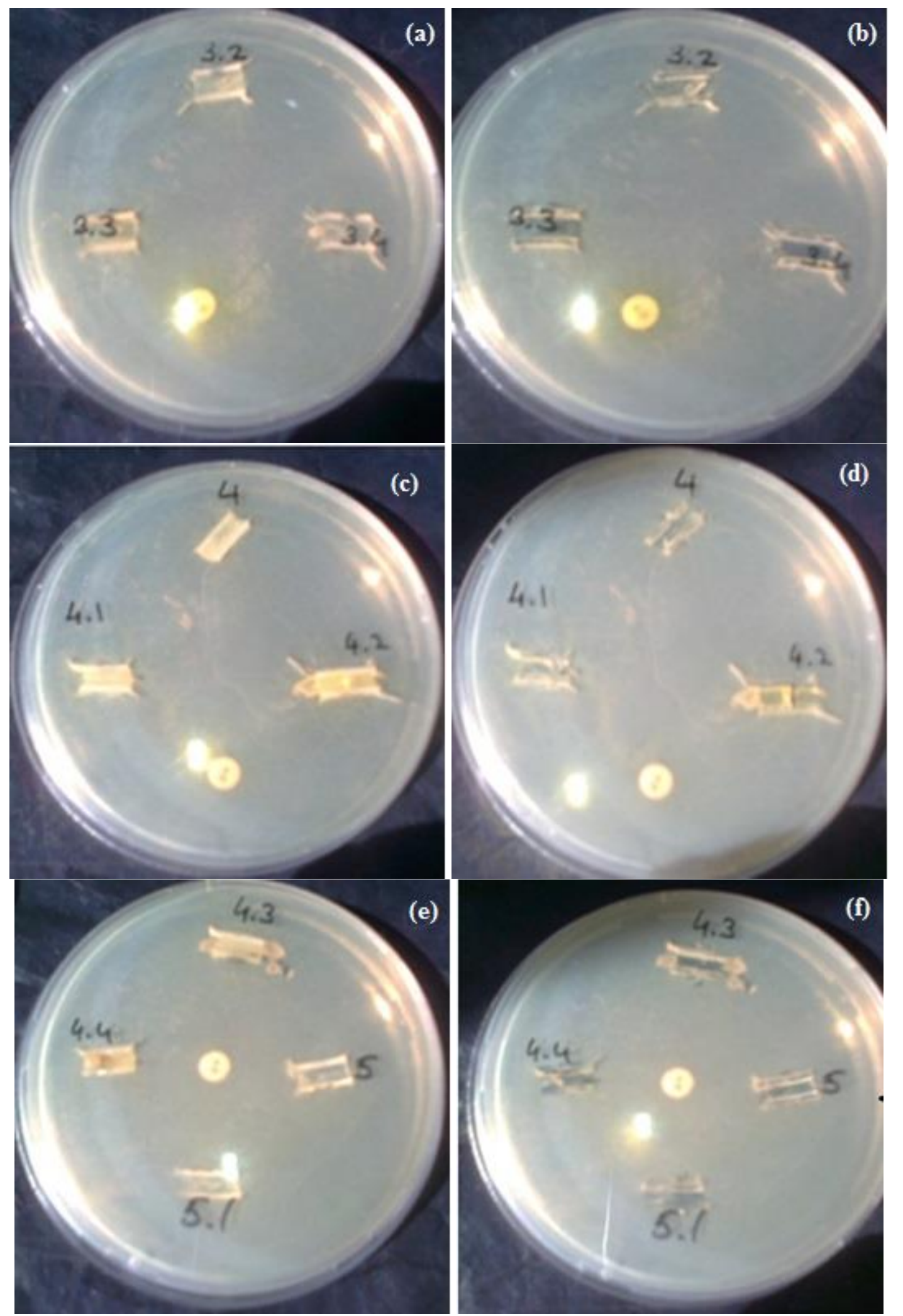

Figure 6. Tubing parts with different diameters $(\mathrm{a}, \mathrm{c}, \mathrm{e})$ and photographs of bacterial growth inhibited areas $(\mathrm{b}$, d, f). Spherical discs: control discs; exp: 4.4 (dialysis tubing type no. modification step)

Figure 7 shows the incubation results of tubing parts of $\sim 2 \mathrm{~mm}$ tested for five nosocomial microorganisms. İnhibition zone formations around samples were observed only for several samples like a bit of zone surrounding them, and again no microbial growth was observed under the samples without zone. It also seems that the type of microorganism affects the antimicrobial zone formation or activity since gram (-) bacterial E. coli showed the maximum resistance coming from the cell wall structure. The wall structures of different 
bacteria exhibit different functional sensitivity [42]. The growth inhibition of AgNPs against E.coli was found more effective than S. aureus, which is also seen in our results of inhibition zone formation as given in Figure $7 \mathrm{a}$.
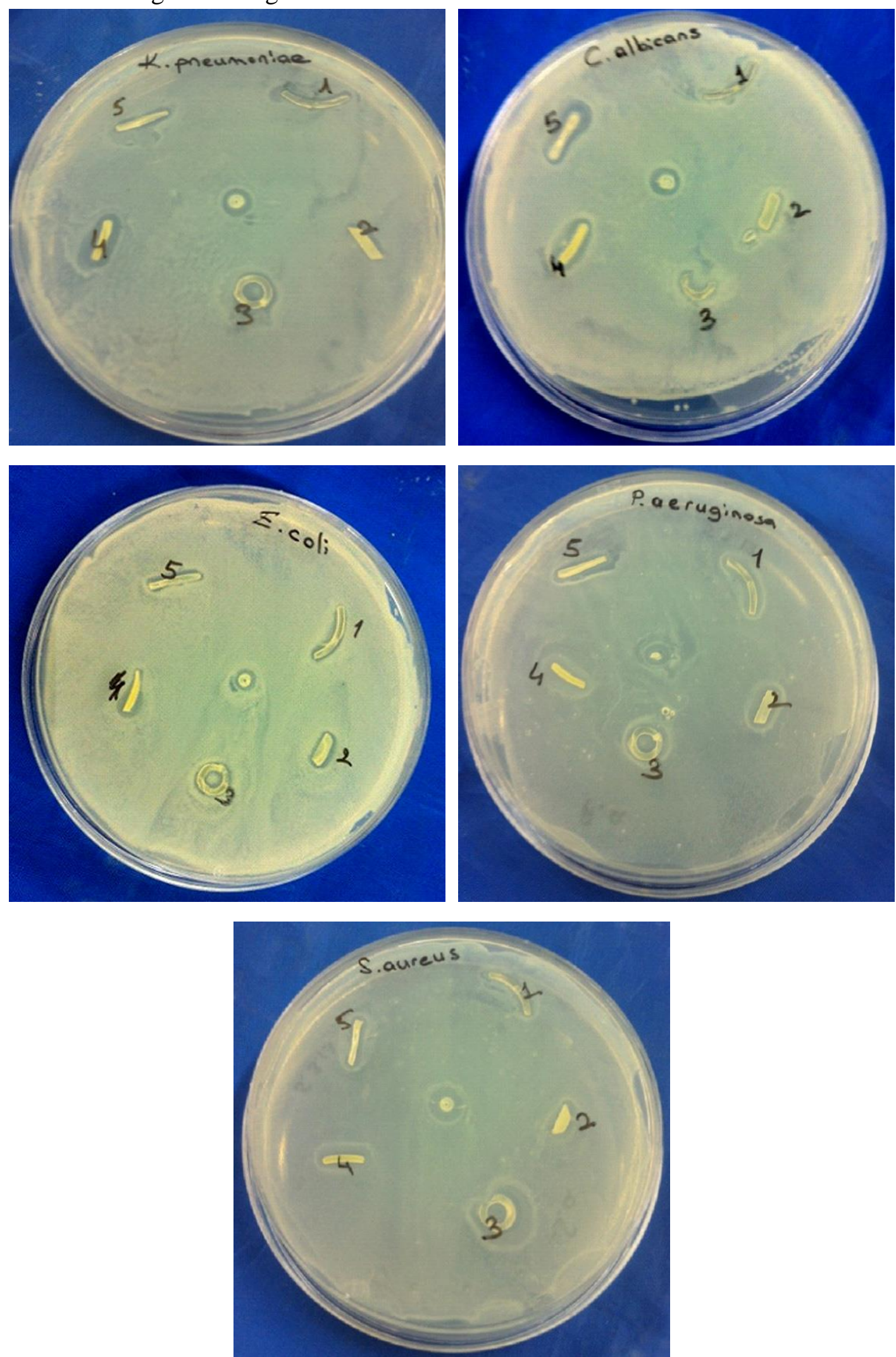

Figure 7. Inhibition zone formation test result of five different tubing diameters for five different microorganisms (types of microorganisms were written on figures).

İnhibition zone formations around samples were observed only for several samples, like a little zone surrounding them. So, another method was used to assure the antimicrobial activity 
on tubings. The tubing parts tested in the first method were cut about $2 \mathrm{~mm}$ width under sterile conditions, placed into the culture tubes inoculated with four bacteria in Nutrient broth $\left(1.2 \times 10^{6}\right.$ $\mathrm{CFU} / \mathrm{mL}$ ) and Sabouraud Dextrose broth with Candida albicans. They were left to incubate at $37^{\circ} \mathrm{C}$ for $24 \mathrm{~h}$ and at $27^{\circ} \mathrm{C}$ for $48-72 \mathrm{~h}$. At the end of the incubation, the blood tubing part in each tube was taken out under sterile conditions, and the culture in tubes was incubated in the same conditions by adding Nutrient agar in order to determine whether a reduction occurred in the number of bacteria/yeast. It was determined at the end of the incubation that no adherence to AgNP immobilized dialysis tubings occurred. The number of bacteria/yeast in culture tubes did not change. Proliferation was not observed for all types of microorganisms. It senses that the surface modification of tubing is not sufficient due to the lack of inhibition zone formation in some samples. There are other important parameters that the tested tubing parts contain both modified and unmodified surfaces due to the thickness of tubing material and the slightly curved shape of tubings prevented to bring out the antimicrobial property. This phenomenon leads to low antimicrobial activity since no microbial adherence and growth were observed in the last stage. Also, the tubing surfaces did not allow to adhere to any microorganism, either bacteria or fungi, which gives a promising result. Also, the microbial growth in culture tubes was not realized, and these phenomena are another positive outcome to prove the success of the method. Microbial proliferation did not occur after treatment with even at a very small modified surface.

There were no important inhibition zone formations for gram (-) and gram (+) bacteria and fungi. It can be concluded that antimicrobial properties were estimated for all types of microorganisms, but the restrictions due to diameter differences of tested tubing materials, it was not possible to measure the right inhibition zones.

Similar behavior was observed for gram-positive anaerobic bacteria, and uncapped AgNPs inhibited more strains. It may be said that the capping of metallic nanoparticles retards their antimicrobial effect to come up due to diffusional restrictions or non-covalent chemical interactions $[37,38,40]$.

The surface of silver nanoparticles was coated with citrate, poly(ethylene glycol), lipoic acid, and tannic acid, and the cytotoxicity was also assessed. PEG-coating was regarded as a good alternative for industrial and medical applications to reduce the toxicity towards human skin cells and gingival fibroblasts $[39,42,43]$.

\section{Conclusions}

In summary, we developed a surface coating strategy by limiting the antimicrobial potential of AgNPs with the presence of PEG on PVC, hemodialysis blood tubings. The extract obtained from leaves of Liquidambar Orientalis Miller was used to synthesize AgNPs with an "Environment-Friendly" approach. The antimicrobial activity against Escherichia coli, Pseudomonas aeruginosa, Klebsiella pneumonia, Staphylococcus aureus, and Candida albicans proved the efficiency of surface-modified extracorporeal tubing biomaterial, which showed promising prospect in extracorporeal blood flow application. Moreover, further research on these methodologies is required to find the release behavior of PEG and AgNPs as well as cytotoxicity of immobilized AgNP. 


\section{Funding}

This work was partly supported by the Scientific Research Projects Coordination Office of Muğla Sitkı Koçman University (Research Project No 16/145) for financial assistance.

\section{Acknowledgments}

The authors are grateful to Prof. Dr. Hakan Ayhan for his help in this research.

\section{Conflicts of Interest}

The authors declare no conflict of interest.

\section{References}

1. Mathew, E.; Domínguez-Robles, J.; Larrañeta, E.; Lamprou, D.A. Fused Deposition Modelling as a Potential Tool for Antimicrobial Dialysis Catheters Manufacturing: New Trends vs. Conventional Approaches Coatings 2019, 9, https://doi.org/10.3390/coatings9080515.

2. Fernandez-Hidalgo, N.; Almirante, B.; Calleja, R.; Ruiz, I.; Planes, A.M.; Rodrugez, D.; Pigrau, C.; Pahissa, A.J. Antibiotic-lock therapy for long-term intravascular catheter-related bacteraemia: results of an open, noncomparative study. J. Antimicrob. Chemother. 2006, 57, 1172-1180, https://doi.org/10.1093/jac/dkl103.

3. Öcal, D.; Dolapçı, I. Santral Venöz Kateter ile İlişkili Enfeksiyonlar. Türk Mikrobiyol Cem Derg. 2012, 42, $1-9$.

4. Li, X.; Huang,T.; Heath, D.E.; O’Brien-Simpson, N.M.; O’Connor, A.J. Antimicrobial nanoparticle coatings for medical implants: Design challenges and prospects. Biointerphases 2020, 15, https://doi.org/10.1116/6.0000625.

5. John, A.; Jernigan, J.; Hatfield, K.M.; Wolford, H.; Nelson, R.E.; Olubajo, B.; Reddy, S.C.; McCarthy, N.; Paul, P.; McDonald, L.C.; Kallen, A.; Fiore, A.; Craig, M.; Baggs, J. Multidrug-Resistant Bacterial Infections in U.S. Hospitalized Patients, 2012-2017. The New England J of Medicine 2020, 382, 1309-1319, https://doi.org/10.1056/NEJMoa1914433.

6. De Freitas, L.W.C.; Neto, M.M.; Nascimento, M.M.P.; Figueiredo, J.F. Bacterial colonization in hemodialysis temporary dual lumen catheters: a propective study. Ren. Fail. 2008, 30, 31-5, https://doi.org/10.1080/08860220701741940.

7. Miller, D.L.; O'Grady, N.P. Guidelines for the Prevention of Intravasculear Catheter-related Infections: Recommendations Relevent to Interventional Radiology for Venous Catheter Placement and Maintanence. J Vasc. Interv. Radiol. 2012, 23, 997-1007, https://doi.org/10.1016/j.jvir.2012.04.023.

8. Wu, K.; Yang, Y.; Zhang, Y.; Deng, J. Lin, C. Antimicrobial activity and cytocompatibility of silver nanoparticles coated catheters via a biomimetic surface functionalization strategy. Int. J. Nanomed. 2015, 10, https://doi.org/10.2147/IJN.S92307.

9. Bayston, R.; Ashraf, W.; Fisher, L. Prevention of infection in neurosurgery: Role of antimicrobial catheters. J. Hosp. Infect. 2007, 65, 39-42, https://doi.org/10.1016/S0195-6701(07)60013-9.

10. Rosenthal, K. Guarding against vascular site infection: Arm yourself with the latest knowledge on equipment and technique to protect patients from catheter-reltated bloodstream infections. Nurs. Manage. 2006, 37, 54-66.

11. Schneider, A.; Baldwin, I.; Souweine, B. What's new: Prevention of acute dialysis catheter-related infection. Intensive Care Med. 2018, 44, 356-358, https://doi.org/10.1007/s00134-017-4833-x.

12. Kranz, J.; Schmidt, S.; Wagenlehner, F.; Schneidewind, L. Catheter-Associated Urinary Tract Infections in Adult Patients. Preventive Strategies and Treatment Options. Dtsch Arztebl Int. 2020, 117, 83-88, https://doi.org/10.3238/arztebl.2020.0083.

13. Fu, J.; Ji, J.; Yuan, W.; Shen, J. Construction of anti-adhesive and antibacterial multilayer films via layerby-layer assembly of heparin and chitosan. Biomaterials 2005, 26, 6684-6692, https://doi.org/10.1016/ j.biomaterials.2005.04.034.

14. Gabriel, G.J.; Som, A.; Madkour, A.E.; Eren, T.; Tew, G.N. İnfectious disease: connecting innate immunity to biocidal polymers. Mater Sci Eng, R. 2007, 57, 28-64, https://doi.org/10.1016/j.mser.2007.03.002.

15. Bertini, G.; Elia, S.; Ceciarini, F.; Dani, C. Reduction of catheter-related bloodstream infections in preterm infants by the use of catheters with the AgION antimicrobial system. Early Hum. Dev. 2013, 89, 21-25, https://doi.org/10.1016/j.earlhumdev.2012.07.003.

16. Natasha, A.;Timotius, K.H. The Biofilm Eradication Using Gentamicin and Anticoagulants as CatheterRelated Infection Prophylaxis in Hemodialysis Patients, Microbiol. Biotechnol. Lett. 2019, 47, 173-182, http://dx.doi.org/10.4014/mbl.1808.08013. 
17. Solis-Castro, R.L.; Silva, L.; Costa, A.; Gebhardt, K.; Cruz, G.J.F. Efficacy of copper-silver ionization for the disinfection of drinking water in Tumbes, Peru. Journal of Physics: Conference Series 2020, 1433, 1-9, https://doi.org/10.1088/1742-6596/1433/1/012011.

18. Liao, C.; Li, Y.; Tjong, S.C. Bactericidal and Cytotoxic Properties of Silver Nanoparticles. Int J Mol Sci. 2019, 20, https://doi.org/10.3390/ijms20020449.

19. Behravan, M.; Panahi, A.H.; Naghizadeh, A.; Ziaee, M.; Mahdavi, R.; Mirzapour, A. Facile green synthesis of silver nanoparticles using Berberis vulgaris leaf and root aqueous extract and its antibacterial activity. Int J of Biol Macromolecules 2019, 124, 148-154, https://doi.org/10.1016/j.ijbiomac.2018.11.101.

20. Divya, K.M.; Raji, P.; Bennet, R.D.; Ratna, G.G.; Kripu, S.V.; Keerthana, D.; Karishma, S.; Antony, V.S.; Paulraj, P.; Thirumurugan, R.; Sajeesh, P.; Jenifer, S.A.; Prakash, P. Green synthesis and antibacterial activity studies of silver nanoparticles from the aqueous extracts of Thespesia populnea. Letters in Applied NanoBioScience. 2020, 9, 931-934, https://doi.org/10.33263/LIANBS91.931934.

21. Kon, K.; Rai, M. Metallic nanoparticles: mechanism of antibacterial action and influencing factors. J.Comp. Clin. Path. Res. 2013, 2, 160-174.

22. Kim, J.S.; Kuk, E.; Yu, K.N.; Kim, J.-H.; Park, S.J.; Lee, H.J.; Kim, S.H.; Park, Y.K.; Park, Y.H.; Hwang, C.-Y.; Kim, Y.-K.; Lee, Y.-S.; Jeong, D.H.; Cho, M.-H. Antimicrobial effects of silver nanoparticles. Nanomedicine: Nanotechnology, Biology and Medicine 2007, 3, 95-101, https://doi.org/10.1016/j. nano.2006.12.001.

23. Sathiyaraj, S.; Suriyakala, G.; Gandhi, A.D.; Saranya, S.; Santhoshkumar, M.; Kavitha, P.; Babujanarthanam, R. Green Biosynthesis of Silver Nanoparticles Using Vallarai Chooranam and Their Potential Biomedical Applications. Journal of Inorganic and Organometallic Polymers and Materials 2020, 30, 4709-4719, https://doi.org/10.1007/s10904-020-01683-7.

24. Rautela, A.; Rani, J.; Debnath, M. Green synthesis of silver nanoparticles from Tectona grandis seeds extract: characterization and mechanism of antimicrobial action on different microorganisms. Journal of Analytical Science and Technology 2019, 10, https://doi.org/10.1186/s40543-018-0163-z.

25. Roe, D.; Karandikar, B.; Bonn-Savage, N.; Gibbins, B.; Roullet, J.-B. Antimicrobial surface functionalization of plastic catheters by silver nanoparticles. Journal of Antimicrobial Chemotherapy 2008, 61, 869-876, https://doi.org/10.1093/jac/dkn034.

26. Guo, L.; Yuan, W.; Lu, Z.; Li, C.M. Polymer/nanosilver composite coatings for antibacterial applications. Colloids and Surfaces A: Physicochemical and Engineering Aspects 2013, 439, 69-83, https://doi.org/10.1016/j.colsurfa.2012.12.029.

27. Roy, A.; Bulut, O.; Some, S.; Mandal, A.K.; Yilmaz, M.D. Green synthesis of silver nanoparticles: biomolecule-nanoparticle organizations targeting antimicrobial activity. RSC Advances 2019, 9, 2673-2702, https://doi.org/10.1039/c8ra08982e.

28. Liao, S.; Zhang, Y.; Pan, X.; Zhu, F.; Jiang, C.; Liu, Q.; Cheng, Z.; Dai, G.; Wu, G.; Wang, L.; Chen, L. Antibacterial activity and mechanism of silver nanoparticles against multidrug-resistant Pseudomonas aeruginosa. Int. J. Nanomedicine. 2019, 14, 1469-1481, https://doi.org/10.2147/ijn.s191340.

29. Fu, J.; Ji, J.; Fan, D.; Shen, J. Construction of antibacterial multilayer films containing nanosilver via layerby-layer assembly of heparin and chitosan-silver ions complex. Journal of biomedical materials research. Part A 2006, 79, 665-674, https://doi.org/10.1002/jbm.a.30819.

30. Ahn, E.-Y.; Jin, H.; Park, Y. Green synthesis and biological activities of silver nanoparticles prepared by Carpesium cernuum extract. Archives of Pharmacal Research 2019, 42, 926-934, https://doi.org/10.1007/s12272-019-01152-x.

31. Manandhar, S.; Luitel, S.; Dahal. R.K. In Vitro Antimicrobial Activity of Some Medicinal Plants against Human Pathogenic Bacteria. J. of Tropical Medicine 2019, 5, https://doi.org/10.1155/2019/1895340.

32. Panpaliya, N.P.; Dahake, P.T.; Kale, Y.J.; Dadpe, M.V.; Kendre, S.B.; Siddiqi, A.G.; Maggavi, U.R. In vitro evaluation of antimicrobial property of silver nanoparticles and chlorhexidine against five different oral pathogenic bacteria. The Saudi Dental Journal 2019, 31, 76-83, https://doi.org/10.1016/j.sdentj. 2018.10.004.

33. Bilgili, B.; Karademir, F.; Bozaci, E.; Özdoğan, E.; Ayhan, H.; Ayhan, F. Liquidambar orientalis mill. Leaf aqueous extract for the synthesis of silver nanoparticles and immobilization on textile fabrics for biomedical applications. Tekstil ve Konfeksiyon 2016, 26, 421-429.

34. Ahmad, S.A.; Nayak, A.K. Bactericidal activity of silver nanoparticles: A mechanistic review. Materials Science for Energy. Technologies 2020, 3, 756-769, https://doi.org/10.1016/j.mset.2020.09.002.

35. Kirtiwar, S.; Gharpure, S.; Balaprasad, A. Effect of Nutrient Media on Antibacterial Activity of Silver Nanoparticles Synthesized Using Neolamarckia cadamba. Journal of Nanoscience and Nanotechnology 2019, 19, 1923-1933, https://doi.org/10.1166/jnn.2019.16117.

36. Mohamad Kasim, A.S.; Ariff, A.B.; Mohamad, R.; Wong, F.W. Interrelations of Synthesis Method, Polyethylene Glycol Coating, Physico-Chemical Characteristics, and Antimicrobial Activity of Silver Nanoparticles. Nanomaterials 2020, 10, https://doi.org/10.3390/nano10122475.

37. Mahato, M.; Pal, P.; Tah, B.; Ghosh, M.; Talapatra, G.B. Study of silver nanoparticle-hemoglobin interaction and composite formation. Colloids Surf B Biointerfaces 2011, 88, 141-149, https://doi.org/10.1016/j.colsurfb.2011.06.024. 
38. Zhang, Z.P.; Rong, M.Z.; Zhang, M.Q.; Yuan, C.e. Alkoxyamine with reduced homolysis temperature and its application in repeated autonomous self-healing of stiff polymers. Polymer Chemistry 2013, 4, 46484654, https://doi.org/10.1039/C3PY00679D.

39. Subhanandaraj, T.T.; Raghavan, K.T.; Narayanan, R. Antibacterial and antibiofilm activity of probiotic based silver nanoparticles is a green approach in biomedical applications. Letters in Applied NanoBioScience. 2020, 9, 988-994, https://doi.org/10.33263/LIANBS92.988994.

40. Ragaseema,V.M.; Rosemary, M.J.; Nandkumar, M.A.; Krishnan, K.V.; Krishnan, L.K. Silver nanoparticle impregnated poly ( $\varepsilon$-caprolactone) scaffolds: optimization of antimicrobial and noncytotoxic concentrations. Tissue Eng. 2011, Part A 17, 439-449, https://doi.org/10.1089/ten.tea.2009.0791.

41. Ragaseema, V.M.; Unnikrishnan, S.; Kalliyana Krishnan, V.; Krishnan, L.K. The antithrombotic and antimicrobial properties of PEG-protected silver nanoparticle coated surfaces. Biomaterials 2012, 33, 30833092, https://doi.org/10.1016/j.biomaterials.2012.01.005.

42. Kim, J.S.; Kuk, E.; Yu, K.N.; Kim, J.-H.; Park, S.J.; Lee, H.J.; Kim, S.H.; Park, Y.K.; Park, Y.H.; Hwang, C.-Y.; Kim, Y.-K.; Lee, Y.-S.; Jeong, D.H.; Cho, M.-H. Antimicrobial effects of silver nanoparticles. Nanomedicine: Nanotechnology, Biology and Medicine 2007, 3, 95-101, https://doi.org/10.1016/j.nano.2006.12.001.

43. Gherasim, O.; Puiu, R.A.; Bîrcă, A.C.; Burdușel, A.-C.; Grumezescu, A.M. An Updated Review on Silver Nanoparticles in Biomedicine. Nanomaterials 2020, 10, https://doi.org/10.3390/nano10112318. 